\title{
Publisher's Note: Study of radiative bottomonium transitions using converted photons [Phys. Rev. D 84, 072002 (2011)]
}

J. P. Lees, V. Poireau, E. Prencipe, V. Tisserand, J. Garra Tico, E. Grauges, M. Martinelli, D. A. Milanes, A. Palano, M. Pappagallo, G. Eigen, B. Stugu, L. Sun, D. N. Brown, L. T. Kerth, Yu. G. Kolomensky, G. Lynch, H. Koch, T. Schroeder,

D. J. Asgeirsson, C. Hearty, T. S. Mattison, J. A. McKenna, A. Khan, V. E. Blinov, A. R. Buzykaev, V. P. Druzhinin,

V. B. Golubev, E. A. Kravchenko, A. P. Onuchin, S. I. Serednyakov, Yu. I. Skovpen, E. P. Solodov, K. Yu. Todyshev, A. N. Yushkov, M. Bondioli, S. Curry, D. Kirkby, A. J. Lankford, M. Mandelkern, D. P. Stoker, H. Atmacan, J. W. Gary,

F. Liu, O. Long, G. M. Vitug, C. Campagnari, T. M. Hong, D. Kovalskyi, J. D. Richman, C. A. West, A. M. Eisner, J. Kroseberg, W. S. Lockman, A. J. Martinez, T. Schalk, B. A. Schumm, A. Seiden, C. H. Cheng, D. A. Doll, B. Echenard, K. T. Flood, D. G. Hitlin, P. Ongmongkolkul, F. C. Porter, A. Y. Rakitin, R. Andreassen, M. S. Dubrovin, B. T. Meadows, M. D. Sokoloff, P. C. Bloom, W. T. Ford, A. Gaz, M. Nagel, U. Nauenberg, J. G. Smith, S. R. Wagner, R. Ayad, W. H. Toki, B. Spaan, M. J. Kobel, K. R. Schubert, R. Schwierz, D. Bernard, M. Verderi, P. J. Clark, S. Playfer, J. E. Watson, D. Bettoni,

C. Bozzi, R. Calabrese, G. Cibinetto, E. Fioravanti, I. Garzia, E. Luppi, M. Munerato, M. Negrini, L. Piemontese,

R. Baldini-Ferroli, A. Calcaterra, R. de Sangro, G. Finocchiaro, M. Nicolaci, S. Pacetti, P. Patteri, I. M. Peruzzi,

M. Piccolo, M. Rama, A. Zallo, R. Contri, E. Guido, M. Lo Vetere, M. R. Monge, S. Passaggio, C. Patrignani, E. Robutti,

B. Bhuyan, V. Prasad, C. L. Lee, M. Morii, A. J. Edwards, A. Adametz, J. Marks, U. Uwer, F. U. Bernlochner, M. Ebert,

H. M. Lacker, T. Lueck, P. D. Dauncey, M. Tibbetts, P. K. Behera, U. Mallik, C. Chen, J. Cochran, H. B. Crawley,

W. T. Meyer, S. Prell, E. I. Rosenberg, A. E. Rubin, A. V. Gritsan, Z. J. Guo, N. Arnaud, M. Davier, D. Derkach,

G. Grosdidier, F. Le Diberder, A. M. Lutz, B. Malaescu, P. Roudeau, M. H. Schune, A. Stocchi, G. Wormser, D. J. Lange,

D. M. Wright, I. Bingham, C. A. Chavez, J. P. Coleman, J. R. Fry, E. Gabathuler, D. E. Hutchcroft, D. J. Payne,

C. Touramanis, A. J. Bevan, F. Di Lodovico, R. Sacco, M. Sigamani, G. Cowan, S. Paramesvaran, D. N. Brown,

C. L. Davis, A. G. Denig, M. Fritsch, W. Gradl, A. Hafner, K. E. Alwyn, D. Bailey, R. J. Barlow, G. Jackson, G. D. Lafferty, R. Cenci, B. Hamilton, A. Jawahery, D. A. Roberts, G. Simi, C. Dallapiccola, E. Salvati, R. Cowan, D. Dujmic, G. Sciolla, D. Lindemann, P. M. Patel, S. H. Robertson, M. Schram, P. Biassoni, A. Lazzaro, V. Lombardo, F. Palombo, S. Stracka,

L. Cremaldi, R. Godang, R. Kroeger, P. Sonnek, D. J. Summers, X. Nguyen, P. Taras, G. De Nardo, D. Monorchio,

G. Onorato, C. Sciacca, G. Raven, H. L. Snoek, C. P. Jessop, K. J. Knoepfel, J. M. LoSecco, W. F. Wang, K. Honscheid,

R. Kass, J. Brau, R. Frey, N. B. Sinev, D. Strom, E. Torrence, E. Feltresi, N. Gagliardi, M. Margoni, M. Morandin,

M. Posocco, M. Rotondo, F. Simonetto, R. Stroili, E. Ben-Haim, M. Bomben, G. R. Bonneaud, H. Briand, G. Calderini,

J. Chauveau, O. Hamon, Ph. Leruste, G. Marchiori, J. Ocariz, S. Sitt, M. Biasini, E. Manoni, A. Rossi, C. Angelini,

G. Batignani, S. Bettarini, M. Carpinelli, G. Casarosa, A. Cervelli, F. Forti, M. A. Giorgi, A. Lusiani, N. Neri, B. Oberhof,

E. Paoloni, A. Perez, G. Rizzo, J. J. Walsh, D. Lopes Pegna, C. Lu, J. Olsen, A. J. S. Smith, A. V. Telnov, F. Anulli,

G. Cavoto, R. Faccini, F. Ferrarotto, F. Ferroni, M. Gaspero, L. Li Gioi, M. A. Mazzoni, G. Piredda, C. Bünger,

T. Hartmann, T. Leddig, H. Schröder, R. Waldi, T. Adye, E. O. Olaiya, F. F. Wilson, S. Emery, G. Hamel de Monchenault,

G. Vasseur, Ch. Yèche, D. Aston, D. J. Bard, R. Bartoldus, J. F. Benitez, C. Cartaro, M. R. Convery, J. Dorfan,

G. P. Dubois-Felsmann, W. Dunwoodie, R. C. Field, M. Franco Sevilla, B. G. Fulsom, A. M. Gabareen, M. T. Graham,

P. Grenier, C. Hast, W. R. Innes, M. H. Kelsey, H. Kim, P. Kim, M. L. Kocian, D. W. G. S. Leith, P. Lewis, S. Li,

B. Lindquist, S. Luitz, V. Luth, H. L. Lynch, D. B. MacFarlane, D. R. Muller, H. Neal, S. Nelson, I. Ofte, M. Perl,

T. Pulliam, B. N. Ratcliff, A. Roodman, A. A. Salnikov, V. Santoro, R. H. Schindler, A. Snyder, D. Su, M. K. Sullivan,

J. Va'vra, A. P. Wagner, M. Weaver, W. J. Wisniewski, M. Wittgen, D. H. Wright, H. W. Wulsin, A. K. Yarritu, C. C. Young,

V. Ziegler, W. Park, M. V. Purohit, R. M. White, J. R. Wilson, A. Randle-Conde, S. J. Sekula, M. Bellis, P. R. Burchat,

T. S. Miyashita, M. S. Alam, J. A. Ernst, R. Gorodeisky, N. Guttman, D. R. Peimer, A. Soffer, P. Lund, S. M. Spanier,

R. Eckmann, J. L. Ritchie, A. M. Ruland, C. J. Schilling, R. F. Schwitters, B. C. Wray, J. M. Izen, X. C. Lou, F. Bianchi,

D. Gamba, L. Lanceri, L. Vitale, N. Lopez-March, F. Martinez-Vidal, A. Oyanguren, H. Ahmed, J. Albert, Sw. Banerjee,

H. H. F. Choi, G. J. King, R. Kowalewski, M. J. Lewczuk, C. Lindsay, I. M. Nugent, J. M. Roney, R. J. Sobie, T. J. Gershon,

P. F. Harrison, T. E. Latham, E. M. T. Puccio, H. R. Band, S. Dasu, Y. Pan, R. Prepost, C. O. Vuosalo, and S. L. Wu

(BABAR Collaboration)

(Received 19 October 2011; published 3 November 2011)

DOI: 10.1103/PhysRevD.84.099901

PACS numbers: 13.20.Gd, 14.40.Pq, 99.10.Fg 
This paper was published online on 10 October 2011 with errors in Table VI and Table VII. The tables have been corrected as of 18 October 2011. The tables are correct in the printed version of the journal. 